\title{
A mouse model of spontaneous preterm birth based on the genetic ablation of biglycan and decorin
}

\author{
Megan L Calmus, Elyse E Macksoud, Richard Tucker, Renato V Iozzo ${ }^{1}$ and Beatrice E Lechner \\ Department of Pediatrics, Women and Infants' Hospital of Rhode Island, The Warren Alpert Medical School of Brown \\ University, 101 Dudley Street, Providence, Rhode Island 02905, USA and ${ }^{1}$ Department of Pathology, Anatomy and \\ Cell Biology, Thomas Jefferson University, 1020 Locust Street, Philadelphia, Pennsylvania 19107, USA
}

Correspondence should be addressed to B E Lechner; Email: blechner@wihri.org

\begin{abstract}
Preterm premature rupture of membranes is responsible for one-third of preterm births. Ehlers-Danlos syndrome (EDS) is associated with preterm premature rupture of membranes in humans. In particular, an EDS variant is caused by a genetic mutation resulting in abnormal secretion of biglycan and decorin, two small leucine-rich proteoglycans highly expressed in reproductive tissues. Because biglycan/decorin null mutant $\left(\mathrm{Bgn}^{-/-} \mathrm{Dcn}^{-/-}\right.$) mice demonstrate phenotypic changes similar to EDS, we used this model to test whether either biglycan or decorin or both play a role in the attainment of successful term gestation. Wild-type biglycan null mutant, decorin null mutant, and biglycan/decorin null mutant pregnancies were assessed for the length of gestation, pup and placenta weight, and litter size. Quantitative real-time PCR was performed to measure biglycan and decorin gene expression, and immunohistochemistry was performed to assess protein expression in placenta and fetal membranes at embryonic days E12, E15, and E18. Bgn ${ }^{-/-} \mathrm{Dcn}^{-/-}$dams $^{-1}$ displayed preterm birth, whereas the possession of at least two biglycan or decorin wild-type alleles was protective of preterm birth. The number of $\mathrm{Bgn}^{-/-} \mathrm{DCn}^{-/-}$pups was decreased at postnatal day P1 but not at E18. Biglycan and decorin were upregulated in the placenta in the absence of each other and were developmentally regulated in fetal membranes, suggesting that these two proteoglycans demonstrate genetic complementation and contribute to gestational success in a dose-dependent manner. Thus, the biglycan/decorin null mutant mouse is a model of genetically induced preterm birth and perinatal loss. This model presents novel targets for preventive or therapeutic manipulation of preterm birth.

Reproduction (2011) 142 183-194
\end{abstract}

\section{Introduction}

Despite significant advances in the care of pregnant mothers and low-birth weight infants, preterm birth is the leading cause of newborn morbidity and mortality and is the main cause of hospitalization in the first year of life in the United States. Moreover, despite numerous interventions, the incidence of prematurity has shown no significant improvement in the last two decades (Ananth \& Vintzileos 2006). Risk factors for prematurity include adverse sociodemographic status, ethnicity, infection, stress, trauma, and a history of a premature birth. However, a majority of preterm births are unexplained (Romero et al. 1994).

Preterm premature rupture of fetal membranes (PPROM) is estimated to account for $40 \%$ of preterm births (Steer 2005). Although recent research has focused on inflammation leading to activation of matrix metalloproteinases (MMPs; Parry \& Strauss 1998, Menon \& Fortunato 2004) as a mechanism, it appears implausible that infection is the only etiologic factor, because the majority of patients have no clinical evidence for an ongoing inflammatory process. Several mechanisms have been proposed to play a role in the process of PPROM, including physical stress, biochemical alterations, and apoptosis (Parry \& Strauss 1998, Menon \& Fortunato 2004, Arikat et al. 2006, El Khwad et al. 2006, Moore et al. 2006). Risk factors for PPROM resemble those for preterm birth and include genetic susceptibility and infection (Parry \& Strauss 1998).

Infants with Ehlers-Danlos syndrome (EDS) have a significantly increased incidence of preterm birth from PPROM in comparison to their unaffected siblings (Barabas 1966, Yen et al. 2006). EDS is a heterogeneous group of rare inherited connective tissue disorders associated with a decrease in tensile strength and integrity of skin, joints, and other connective tissues.

In patients afflicted with the progeroid variant of EDS, the molecular basis of the connective tissue anomaly is a mutation of xylosylprotein-4 $\beta$-galactosyl-transferase I, an enzyme that is necessary for the posttranslational glycosylation of biglycan and decorin, two small leucine-rich proteoglycans (SLRPs; Schaefer \& lozzo 2008) that are involved in regulating collagen 
fibrillogenesis, cell growth, and inflammatory responses (Reed \& lozzo 2002). This mutation leads to the abnormal secretion of biglycan and decorin core protein lacking glycosaminoglycan side chains (Kresse et al. 1987, Quentin et al. 1990).

Mice deficient in biglycan, decorin, or both model the phenotype of EDS, displaying connective tissue anomalies of skin, bone, and tendon (Danielson et al. 1997, Xu et al. 1998).

Biglycan is a SLRP that is a component of the extracellular matrix in various tissues including skin, bone, tendon, and connective tissues (Young et al. 2002, Wadhwa et al. 2004). Its core protein contains two chondroitin or dermatan sulfate side chains (Bowe et al. 2000). Biglycan binds to collagen VI, transforming growth factor $\alpha$ (TGFA) and $\beta$ (TGFB), chordin, and bone morphogenetic protein 4 (BMP4; Hildebrand et al. 1994, Wiberg et al. 2001, 2003, Chen et al. 2004, Hayashi et al. 2005, Moreno et al. 2005). Decorin is an SLRP protein with one chondroitin or dermatan sulfate side chain that demonstrates $\sim 55 \%$ homology with biglycan (Iozzo \& Murdoch 1996) and also interacts with a number of extracellular matrix constituents and growth factors (lozzo \& Schaefer 2010).

Biglycan and decorin are expressed in various gestational tissues in humans and mice (San Martin \& Zorn 2003, San Martin et al. 2003, Chen et al. 2007). They are expressed in the human placenta, where they are increased in association with gestational diabetes mellitus (Chen et al. 2007). Biglycan is expressed in the uterus of pregnant mouse (San Martin \& Zorn 2003, San Martin et al. 2003), while both biglycan and decorin are decreased in the human myometrium during labor (Hjelm et al. 2002). In decorin homozygous null mutant mice, the decidualized stroma of the uterus shows abnormal architecture with large diameters and irregular contours of the endometrial collagen fibrils (Sanches et al. 2010). Furthermore, these SLRPs are the most abundant proteoglycans expressed in human fetal membranes (Gogiel \& Jaworski 2000, Meinert et al. 2001, Gogiel et al. 2003, Valiyaveettil et al. 2004). After labor, biglycan increases, whereas decorin decreases in fetal membranes (Meinert et al. 2007). However, little is known about the role these proteoglycans play in the maintenance of gestation.

Thus, we hypothesize that biglycan and decorin are necessary for the attainment of successful full-term gestation and that the absence of both biglycan and decorin will lead to preterm birth.

\section{Results}

First, we evaluated the reproductive capacity of the following females compared to the wild type $\left(\mathrm{Bgn}^{+/+} \mathrm{DCn}^{+/+}\right)$: biglycan homozygous null mutant and decorin homozygous null mutant mice $\left(B g n^{-1-} \mathrm{DCn}^{+/+}\right.$ and $\left.\mathrm{Bgn}^{+/+} \mathrm{DCn}^{-/-}\right)$, the biglycan heterozygous/decorin heterozygous null mutant $\left(\mathrm{Bgn}^{+/-} \mathrm{Dcn}^{+/-}\right)$, the biglycan heterozygous/decorin homozygous null mutant $\left(\mathrm{Bgn}^{+/-} \mathrm{DCn}^{-/-}\right)$, the biglycan homozygous/decorin heterozygous null mutant $\left(\mathrm{Bgn}^{-1-} \mathrm{DCn}^{+-}\right)$, and the biglycan homozygous/decorin homozygous null mutant $\left(B g n^{-1-} D C n^{-1-}\right)$. Wild-type females, biglycan homozygous null mutant females, and decorin homozygous null mutant females were bred with males of their own genotype. $\mathrm{Bgn}^{+/-} \mathrm{DCn}^{+/-}, \mathrm{Bgn}^{+/-} \mathrm{DCn}^{-/-}$, $\mathrm{Bgn}^{-/-} \mathrm{Dcn} \mathrm{n}^{+/-}$, and $\mathrm{Bgn}^{-1-} \mathrm{Dcn}^{-1-}$ females were bred with $\mathrm{Bgn}^{-10} \mathrm{Dcn}^{+/-}$males as described in the Materials and Methods section.

When comparing mating frequency (defined as the number of days of mating necessary for the formation of a plug that leads to clinically apparent pregnancy), there were no differences among $\mathrm{Bgn}^{-/-} \mathrm{DCn}^{+/+}$, $\mathrm{Bgn}^{+/+} \mathrm{DCn}^{-1-}$, and $\mathrm{Bgn}^{-1-} \mathrm{Dcn}{ }^{-9-}$ dams compared with $\mathrm{Bgn}^{+/+} \mathrm{DCn}^{+/+}$dams $(P=0.195$; Supplementary Figure $1 \mathrm{~A}$, see section on supplementary data given at the end of this article). The rate of progression from plugging to viable pregnancy demonstrated a significant decrease in the $\mathrm{Bgn}^{+/+} \mathrm{Dcn}^{-1-}$ and $\mathrm{Bgn}^{-1-} \mathrm{Dcn}^{-1-}$ dams but not in $\mathrm{Bgn}^{-/-} \mathrm{DCn}^{+/+}$dams compared with $\mathrm{Bgn}^{+/+} \mathrm{DCn}^{+/+}$dams $(P=0.045, P=0.022$, and $P=0.153$ respectively; Supplementary Figure $1 \mathrm{~B})$. Comparing prenatal litter size as well as number of viable versus resorbing embryo implantation sites at E12, E15, and E18, we observed no significant difference in $\mathrm{Bgn}^{-/-} \mathrm{DCn}^{+/+}$and $\mathrm{Bgn}^{+/+} \mathrm{DCn}^{-1-}$ dams compared with $\mathrm{Bgn}^{+/+} \mathrm{Dcn}^{+/+}$dams $(P=0.574$ and $P=0.385$ respectively; Supplementary Figure $2 \mathrm{~A}$ and $\mathrm{B}$, see section on supplementary data given at the end of this article).

We observed that the $\mathrm{Bgn}^{-/-} \mathrm{Dcn}^{+/+}, \mathrm{Bgn}^{+/+} \mathrm{Dcn}^{-1-}$, $\mathrm{Bgn}^{+/-} \mathrm{DCn}^{+/-}$, as well as the wild-type females did not display preterm birth (defined as birth before E18). The $\mathrm{Bgn}^{+}-\mathrm{DCn}^{-1-}$ and the $\mathrm{Bgn}^{-1-} \mathrm{Dcn}^{+/-}$females had a significantly increased risk of preterm birth compared with the wild type, whereas the $B g n^{-1-} D \mathrm{Cn}^{-1-}$ had further significantly increased risk of preterm birth (Fig. 1; $P=0.0002$ ). This increase in the rate of preterm birth displayed an inversely linear relationship to the number of SLRP (biglycan or decorin) alleles per genotype (Table 1; $P<0.0001)$. Furthermore, we observed that the $\mathrm{Bgn}^{-1-} \mathrm{DCn}^{-1-}$ dams displayed a significantly decreased gestational length (Fig. 2A; $P<0.001$ ) as well as a significantly reduced litter size (defined as pups surviving the first $24 \mathrm{~h}$ after birth) compared with the control wild type (Fig. 2B; $P<0.001$ ).

In mixed litters of $\mathrm{Bgn}^{+/-} \mathrm{Dcn}^{+/-}$females mated with $\mathrm{Bgn}^{-10} \mathrm{Dcn}^{+/-}$males, the mean litter size significantly decreased from E18 to P1 (Table 2). Concurrently, the number of biglycan homozygous/decorin homozygous null mutant $\left(\mathrm{Bgn}^{-1-} \mathrm{DCn^{-1 }}\right)$ pups was as expected by Mendelian calculations at E18 but was significantly lower than expected at P1, suggesting a perinatal loss of $B g n^{-1-} D C n^{-1-}$ pups as a cause of the decrease in litter size (Table 2; $P<0.001$ ). Furthermore, $B g n^{-1-} D c n^{-1-}$ 


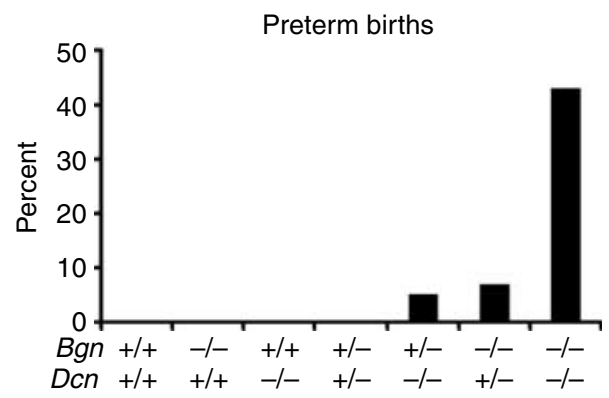

Figure 1 Percentage of preterm births (before E18) per mouse genotype. Biglycan and decorin are necessary for the maintenance of gestation to full term in a dose-dependent and compensatory manner. Biglycan homozygous null mutants, decorin homozygous null mutants, and biglycan heterozygous/decorin heterozygous null mutant are not at increased risk of preterm birth. Biglycan heterozygous/decorin homozygous null mutants and biglycan homozygous/decorin heterozygous null mutants are at increased risk of preterm birth, whereas biglycan homozygous/decorin homozygous null mutants are at further increased risk of preterm birth, $P=0.0002 . \chi^{2}$ test: $\mathrm{Bgn}^{+/+} \mathrm{DCn}^{+/+}$, $n=13 ; \mathrm{Bgn}^{-/-} \mathrm{Dcn}^{+/+}, n=33 ; \mathrm{Bgn}^{+/+} \mathrm{Dcn}^{-/-}, n=11$; $\mathrm{Bgn}^{+/}-\mathrm{DCn}^{+/-}, \mathrm{n}=14 ; \mathrm{Bgn}^{+/-} \mathrm{DCn}^{-1-}, n=20 ; \mathrm{Bgn}^{-/-} \mathrm{DCn}^{+/-}$, $n=30 ; B g n^{-1-} \mathrm{CCn}^{-1-}, n=7$. Bgn, biglycan; DCn, decorin.

pups displayed lower weights at P1 than their littermate controls and displayed a trend toward lower weights at E18 that was not statistically significant, although their placental weights at E18 were not different from littermate controls (Fig. 3A-C; $P=0.0014, P=0.2068$, and $P=0.858$ respectively).

As a first step in pinpointing the compensatory mechanisms that allow for normal gestational phenotypes in null mutants that possess at least two of four wildtype SLRP alleles, we performed immunohistochemical analysis of gestational tissues at E18. We assessed decorin expression and localization in the placenta and fetal membranes in the presence and absence of biglycan, as well as the expression and localization of biglycan in the same tissues in the presence and absence of decorin. Each experiment was repeated three times. Comparing placental sections of wild-type and biglycan homozygous null mutant mice, we noted a robust upregulation of decorin in the absence of biglycan. Conversely, biglycan was upregulated in the decorin homozygous null mutant placenta (Fig. 4A). We did not note a change in biglycan expression in decorin

Table 1 The percentage of preterm mouse births $(<\mathrm{E} 18)$ increases with decreasing number of maternal biglycan and decorin alleles.

\begin{tabular}{lcc}
\hline SLRP alleles & Preterm births* & Total births \\
\hline 4 & $0(0 \%)$ & 13 \\
2 & $0(0 \%)$ & 55 \\
1 & $3(6 \%)$ & 50 \\
0 & $3(43 \%)$ & 7
\end{tabular}

${ }^{*} \chi^{2}$ for linear trend test: $P<0.0001$. SLRP, small leucine rich proteoglycan. homozygous null mutant fetal membranes or in decorin expression in biglycan homozygous null mutant fetal membranes. Our results were similar for all tissue types at E12 and E15, but the biglycan and decorin upregulation in the placenta was less striking than that at E18 (data not shown). Quantitative analysis revealed a statistically significant increase in decorin immunofluorescence in the biglycan homozygous null mutant placenta compared with the wild type $(P<0.001)$, as well as a statistically significant increase in biglycan immunofluorescence in the decorin homozygous null mutant $(P<0.001)$, but no difference in fetal membranes (Fig. 4B).

Next, in order to assess whether the protein compensation between biglycan and decorin in the placenta was also present at the transcriptional level, we quantified gene expression of these SLRPs in mouse gestational tissues during the course of gestation. We measured biglycan mRNA levels in the wild-type and decorin homozygous null mutant and decorin mRNA levels in the wild-type and biglycan homozygous null mutant in placenta and fetal membranes at E12, E15, and E1 8 via quantitative real-time PCR. Each experiment was repeated three times. Our data indicate that biglycan is developmentally regulated in fetal membranes $(P=0.017)$ and placenta $(P=0.005)$, and decorin is developmentally regulated in fetal membranes $(P<0.001)$ but not in the placenta $(P=0.188)$. However, there is no significant difference in biglycan transcript levels in the presence or absence of decorin in either of the gestational tissue types. Likewise, there is no difference in decorin transcript levels in the presence or absence of biglycan in either of the gestational tissue types (Fig. 5).

\section{Discussion}

To our knowledge, this is the first established mouse model of a genetic mechanism of spontaneous preterm birth. In other models of preterm birth, for example, the interleukin-18, interleukin-10, and recombination activating gene 1 knockout models, preterm birth was linked to an inflammatory insult and was not spontaneous (Robertson et al. 2006, Wang et al. 2006, Bizargity et al. 2009, Thaxton et al. 2009). Furthermore, to our knowledge, this is the first mouse model of genetic preterm birth that displays a correlation to a human disease associated with preterm birth.

The underlying cause of the progeroid variant of EDS, a disorder associated with decreased connective tissue tensile strength, is a mutation in the enzyme xylosylprotein-4 $\beta$-galactosyl-transferase I (Kresse et al. 1987, Quentin et al. 1990). This mutation prevents posttranslational glycosylation of biglycan and decorin, resulting in the secretion of non-glycosylated abnormal biglycan and decorin protein cores (Kresse et al. 1987, Quentin et al. 1990). Infants born with EDS have a significantly 

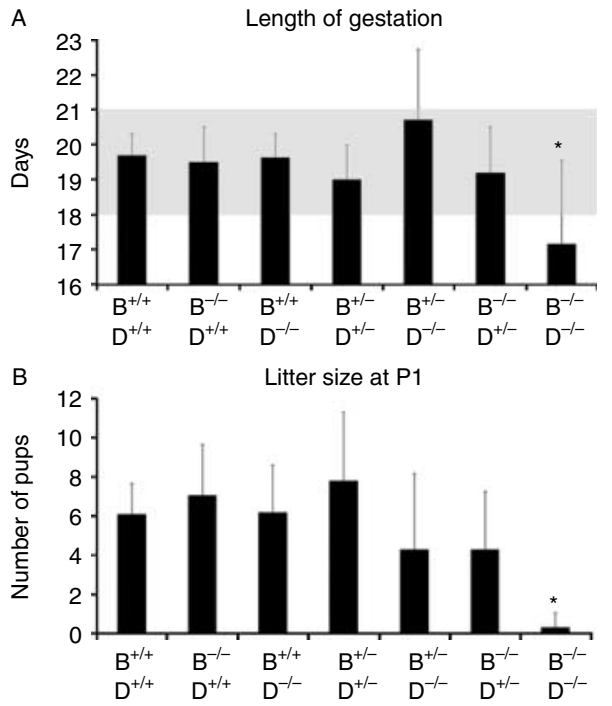

Figure 2 (A) $\mathrm{Bgn}^{-1-} \mathrm{DCn}^{-1-}$ female mice display a statistically significant decrease in length of gestation compared with all other genotypes, $P<0.001 . \mathrm{Bgn}^{+/+} \mathrm{DCn}^{+/+}, n=13 ; \mathrm{Bgn}^{-/-} \mathrm{DCn}^{+/+}$, $n=33 ; \mathrm{Bgn}^{+/+} \mathrm{DCn}^{-/-}, n=11 ; \mathrm{Bgn}^{+/-} \mathrm{DCn}^{+/-}, n=13$;

$\mathrm{Bgn}^{+/-} \mathrm{DCn}^{-1-}, n=14 ; \mathrm{Bgn}^{-1-} \mathrm{DCn}^{+/-}, n=20 ; \mathrm{Bgn}^{-/-} \mathrm{DCn}^{-1-}$, $n=7$. Shading: range of normal length of pregnancy. (B) Number of mouse pups per litter surviving beyond $24 \mathrm{~h}$ of age. $\mathrm{Bgn}^{-1-} \mathrm{DCn} \mathrm{C}^{-1-}$ dams display a significantly decreased litter size at $24 \mathrm{~h}, P<0.001$. $\mathrm{Bgn}^{+/+} \mathrm{DCn}^{+/+}, \mathrm{n}=11 ; \mathrm{Bgn}^{-1-} \mathrm{DCn}^{+/+}, n=17 ; \mathrm{Bgn}^{+/+} \mathrm{DCn}^{-1-}$, $n=32 ; \mathrm{Bgn}^{+/-} \mathrm{DCn}^{+/-}, n=54 ; \mathrm{Bgn}^{+/-} \mathrm{DCn}^{-/-}, n=34$;

$\mathrm{Bgn}^{-1-} \mathrm{DCn}^{+/-}, n=19 ; \mathrm{Bgn}^{-1-} \mathrm{DCn}^{-1-}, n=7$. Error bars=s.E.M. $\mathrm{P}$, postnatal day; Bgn, biglycan; Dcn, decorin. One-way ANOVA with Holm-Sidak contrast was performed for both sets of data $(* P<0.05)$.

elevated incidence of preterm birth from PPROM in comparison to their unaffected siblings (Barabas 1966). Thus, the biglycan homozygous/decorin homozygous null mutant mouse $\left(\mathrm{Bgn}^{-1-} \mathrm{DCn}^{-1-}\right)$ is a novel model of human preterm birth caused by genetic predisposition.

The significance of this model extends beyond its utility as a model for preterm birth secondary to the rare EDS. Otherwise healthy women with a history of recurrent preterm birth secondary to PPROM of unknown etiology have skin morphology similar to patients with EDS (Hermanns-Le et al. 2005). In women with recurrent PPROM, the histochemical structure of the dermis showed thin, irregularly shaped collagen bundles with little interconnectivity (Hermanns-Le et al. 2005). This finding suggests the existence of a spectrum of clinical presentations in which EDS with its full scope of connective tissue abnormalities may represent the extreme form and phenotypically healthy women with recurrent PPROM represent the mild end of the spectrum. Mutations in biglycan and decorin may be part of undiagnosed predisposing genetic factors for preterm birth. Hence, our model has the potential to provide insight into novel mechanisms that predispose phenotypically healthy women to 'idiopathic' PPROM.

Our studies indicate that biglycan and decorin contribute to the attainment of full-term gestation in a gene dose-dependent manner. The loss of two of the four possible wild-type SLRP alleles in the dam does not change the attainment of full-term gestation when compared with the wild-type dam. However, the loss of three wild-type alleles, and even more dramatically the loss of all four wild-type alleles, results in a significantly increased risk of preterm birth. These observations suggest that the complete absence of both biglycan and decorin wild-type alleles results in dysregulation of processes that are protective of preterm birth.

Decorin plays a role in trophoblast migration (Xu et al. 2002, lacob et al. 2008). In the placenta, we observed an increase in biglycan expression in the absence of decorin and an increase in decorin expression in the absence of biglycan. Thus, we suggest that the appropriate birth weight in the biglycan homozygous null mutant and decorin homozygous null mutant compared with the biglycan homozygous/decorin homozygous null mutant female as well as successful gestational outcomes are a function of compensatory SLRP upregulation in the placenta. This is an indication of some functional overlap between the two proteoglycans. Furthermore, the attainment of full-term gestation seen in the biglycan homozygous null mutant, decorin homozygous null mutant, and the biglycan heterozygous/decorin

Table 2 The mean litter size for $B g n^{+/-} D c n^{+/-}$dams is decreased at P1 secondary to the loss of $B g n^{-/-} D c n^{-1-}$ mouse pups

\begin{tabular}{|c|c|c|c|}
\hline Genotype & E18 $(n)$ & $\mathbf{P 1}(n)$ & Mendelian expected $(\%)$ \\
\hline \multicolumn{4}{|c|}{ (A) The mean litter size for $\mathrm{Bgn}^{+/-} \mathrm{Dcn}^{+/-}$dams is decreased at P1 compared with E18 } \\
\hline $\mathrm{Bgn}^{+1-} \mathrm{DCn}^{+1-}-\mathrm{Con}$ & $9.3(10)$ & $6.6(14)^{*}$ & \\
\hline \multicolumn{4}{|c|}{ (B) The number of $\mathrm{Bgn}^{-1-} \mathrm{DCn}^{-1-}$ mouse pups is unchanged prenatally but decreased postnatally compared with Mendelian distribution } \\
\hline $\mathrm{Bgn}^{+/ 0} \mathrm{Dcn}^{+/+}$ & $5 \%(5)$ & $7 \%(10)$ & 6.25 \\
\hline $\mathrm{Bgn}^{+/ 0} \mathrm{DCn}^{+/-}$ & $17 \%(16)$ & $14 \%(19)$ & 12.50 \\
\hline $\mathrm{Bgn}^{+/ 0} \mathrm{DCn}^{-1-}$ & $5 \%(5)$ & $4 \%(5)$ & 6.25 \\
\hline $\mathrm{Bgn}^{+/-} \mathrm{DCn}^{+/+}$ & $9 \%(8)$ & $12 \%(16)$ & 6.25 \\
\hline $\mathrm{Bgn}^{+/-} \mathrm{DCn}^{+/-}$ & $12 \%(11)$ & $23 \%(31)$ & 12.50 \\
\hline $\mathrm{Bgn}^{+/-} \mathrm{DCn}^{-1-}$ & $6 \%(6)$ & $6 \%(8)$ & 6.25 \\
\hline $\mathrm{Bgn}^{-1-} \mathrm{Dcn}^{+/+}$or $\mathrm{Bgn}^{-10} \mathrm{Dcn}^{+/+}$ & $8 \%(7)$ & $10 \%(13)$ & 12.50 \\
\hline $\mathrm{Bgn}^{-1-} \mathrm{DCn}^{+/-}$or $\mathrm{Bgn}^{-10} \mathrm{DCn}^{+/-}$ & $22 \%(20)$ & $21 \%(28)$ & 25 \\
\hline $\mathrm{Bgn}^{-1-} \mathrm{DCn}^{-1-}$ or $\mathrm{Bgn}^{-10} \mathrm{DCn}^{-1-}$ & $16 \%(15)$ & $3 \%(4)^{+}$ & 12.50 \\
\hline
\end{tabular}

${ }^{*} P<0.05 ;{ }^{\dagger} P<0.001$. 


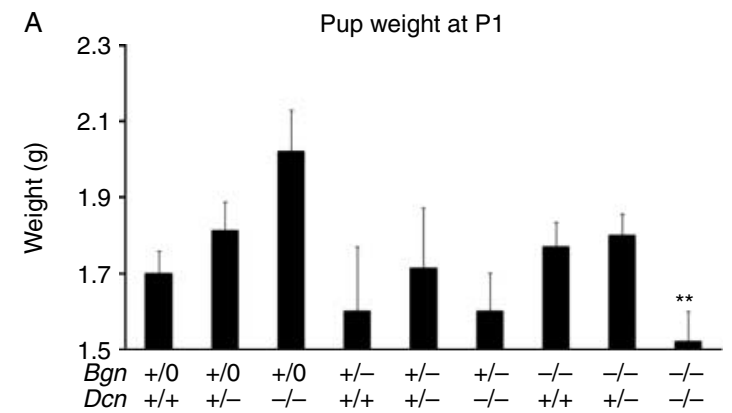

$\mathrm{B}$

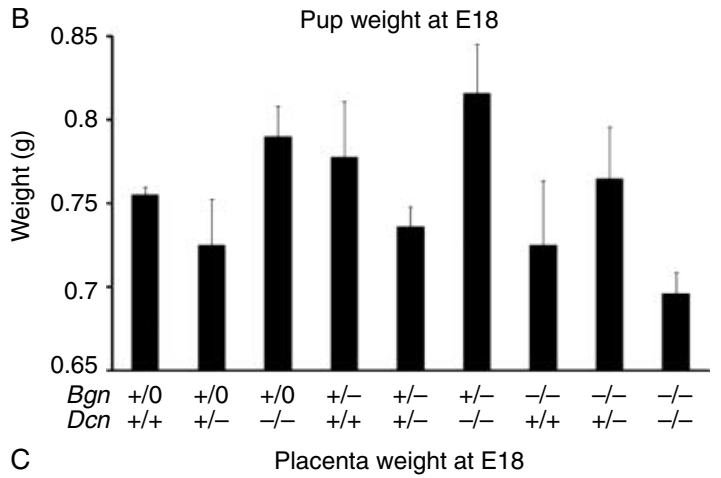

C Placenta weight at E18

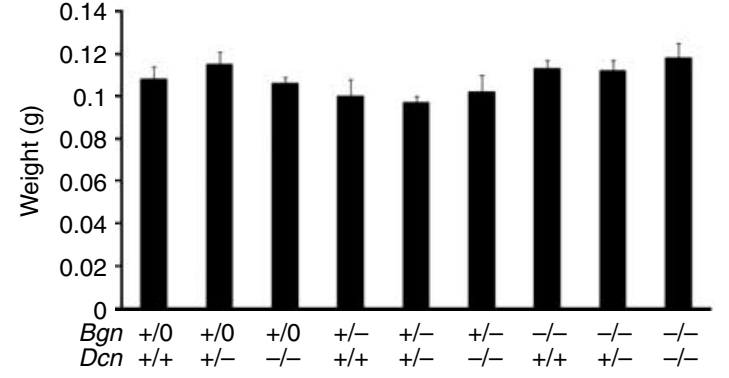

Figure 3 (A) Biglycan heterozygous/decorin heterozygous null mutant mouse dams' litters were weighed on P1. Bgn ${ }^{-1-} \mathrm{DCn}^{-1-}$ pups significantly display lower weight compared with littermates of other genotypes, $P=0.0014$. Pregnant $B g n^{+/-} D c n^{+/-}$dams, $n=12$; litters, $n=14$; total pups in litters, $n=93 ; \mathrm{Bgn}^{+/+} \mathrm{DCn}^{+/+}, n=7$;

$\mathrm{Bgn}^{+/+} \mathrm{DCn}^{+/-}, n=16 ; \mathrm{Bgn}^{+/+} \mathrm{DCn}^{-1-}, n=14 ; \mathrm{Bgn}^{+/-} \mathrm{DCn}^{+/+}$ $n=4 ; B g n^{+/-} \mathrm{DCn}^{+/-}, n=7 ; \mathrm{Bgn}^{+/-} \mathrm{DCn}^{-/-}, n=2 ; \mathrm{Bgn}^{-/-} \mathrm{DCn}^{+/+}$, $n=13 ; \mathrm{Bgn}^{-/-} \mathrm{DCn}^{+/-}, n=25 ;$ and $\mathrm{Bgn}^{-/-} \mathrm{DCn}^{-/-}, n=5$.

(B) Biglycan heterozygous/decorin heterozygous null mutant mouse dams' litters were weighed on E18. As opposed to P1 pup weights, there is no significant difference in pup weight at E18 between littermates of various genotypes. Pregnant $B g n^{+/-} D C n^{+/-}$dams, $n=7$; total pups in litters, $n=63 ; B g n^{+/+} D_{C n}{ }^{+/+}, n=2 ; B g n^{+/+} D_{C n} n^{+/-}, n=8$;

$\mathrm{Bgn}^{+/+} \mathrm{DCn}^{-/-}, n=5 ; \mathrm{Bgn}^{+/-} \mathrm{DCn}^{+/+}, n=9 ; \mathrm{Bgn}^{+/-} \mathrm{DCn}^{+/-}, n=8$; $\mathrm{Bgn}^{+/-} \mathrm{DCn}^{-1-}, n=5 ; \mathrm{Bgn}^{-1-} \mathrm{DCn}^{+1+}, n=6 ; \mathrm{Bgn}^{-1-} \mathrm{DCn}^{+1-}$, $n=15$; and $B g n^{-1-} D C n^{-1-}, n=5$. (C) Biglycan heterozygous/decorin heterozygous null mutant mouse dams' placenta weight was measured on E18. As opposed to P1 pup weights, there is no significant difference in placenta weight between littermates of various genotypes. Pregnant $B g n^{+/-} \mathrm{DCn}^{+/-}$dams, $n=10$; total pups in litters, $n=93$; $\mathrm{Bgn}^{+/+} \mathrm{DCn}^{+/+}, n=8 ; \mathrm{Bgn}^{+/+} \mathrm{DCn}^{+/-}, n=11 ; \mathrm{Bgn}^{+1+} \mathrm{DCn}^{-1-}$, $n=6 ; \mathrm{Bgn}^{+/-} \mathrm{DCn}^{+/+}, n=5 ; \mathrm{Bgn}^{+/-} \mathrm{DCn}^{+/-}, n=16 ; \mathrm{Bgn}^{+/-}$ $\mathrm{DCn}^{-1-}, n=5 ; \mathrm{Bgn}^{-1-} \mathrm{DCn}^{+/+}, n=7 ; \mathrm{Bgn}^{-1-} \mathrm{DCn}^{+/-}, n=20$; and $\mathrm{Bgn}^{-1-} \mathrm{DCn}^{-/-}, n=15 . \mathrm{Bgn}^{-/-}$refers to $\mathrm{Bgn}^{-1-}$ or $\mathrm{Bgn}^{-/ 0}$ depending on gender. Error bars=s.E.M. P, postnatal day; Bgn, biglycan; Dcn, decorin. Mixed statistical model: genotype as a fixed effect, litter as a random effect, specific contrasts of $\mathrm{Bgn}^{-1-} \mathrm{DCn}^{-1-}$ versus all other genotypes $(* * P<0.01)$. heterozygous null mutant $\left(\mathrm{Bgn}^{-/-} \mathrm{Dcn} \mathrm{n}^{+/+}\right.$, $\mathrm{Bgn}^{+/+} \mathrm{DCn}^{-/-}$, and $\mathrm{Bgn}^{+/-} \mathrm{DCn}^{+/-}$) compared with the impaired preterm birth reproductive phenotype of the biglycan heterozygous/decorin homozygous null mutant, the biglycan homozygous/decorin heterozygous null mutant, and the biglycan homozygous/decorin homozygous null mutant $\left(\mathrm{Bgn}^{-1-} \mathrm{DCn}^{++-}, \mathrm{Bgn}^{+/-}\right.$ $D \mathrm{Cn}^{-1-}$ and $B g n^{-1-} D \mathrm{Cn}^{-1-}$ ) may also be a function of compensatory SLRP upregulation. This ability to compensate for each other in reproductive tissues is similar to the genetic compensation that has been reported in skin, bone, muscle, kidney, and cornea (Ameye \& Young 2002, Corsi et al. 2002, Zhang et al. 2009) between biglycan and decorin, as well as in tendon between another set of SLRPs, lumican and fibromodulin (Svensson et al. 1999). Interestingly, we did not observe similar compensatory upregulation of protein expression in the fetal membranes. This could be a result of compensatory mechanisms not being present in the fetal membranes. Alternately, it may be a reflection of the fact that given the abundance of both biglycan and decorin in fetal membranes (Parry \& Strauss 1998), a possible compensatory increase may not be appreciable with the techniques used.

The compensatory biglycan and decorin protein upregulation we observed in the placenta of decorin homozygous null mutant and biglycan homozygous null mutant dams was not present at the gene transcript level, suggesting that the compensation is secondary to posttranscriptional processes such as increased mRNA stabilization or decreased protein degradation and not increased gene expression. Also, we observed that both biglycan and decorin are developmentally regulated in fetal membranes and biglycan, but not decorin, is developmentally regulated in the placenta. This again suggests an important role in the maintenance of successful gestation, as well as underlining that biglycan and decorin act via discrete mechanisms.

Biglycan homozygous/decorin homozygous null mutant $\left(\mathrm{Bgn}^{-1-} \mathrm{DCn}^{--}\right)$pups have lower birth weights and a trend toward lower late gestational (E18) weights than their littermate controls but not lower placental weights. This suggests that the absence of both biglycan and decorin in the pup leads to in utero growth restriction or, conversely, that having at least partial biglycan or decorin competence is protective for appropriate fetal and early postnatal growth. However, biglycan and decorin are not necessary for appropriate placental growth. This observation is consistent with infants born with EDS, who display low birth weight (Roop \& Brost 1999) as well as with infants with in utero growth restriction, who display decreased placental biglycan and decorin expression (Murthi et al. 2010, Swan et al. 2010). Given that mean litter size decreases between E18 and P1 and subsequently less than the Mendelian expected numbers of biglycan homozygous/ decorin homozygous null mutant $\left(\mathrm{Bgn}^{-/-} \mathrm{DCn}^{-/-}\right)$pups 
A

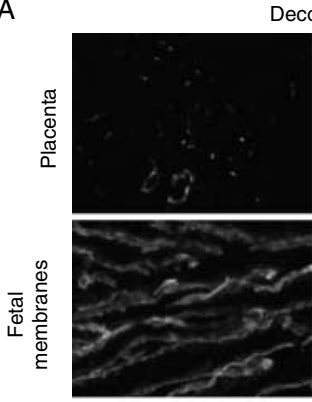

$\mathrm{Bgn}^{+/+} \mathrm{Dcn}^{+/+}$ corin
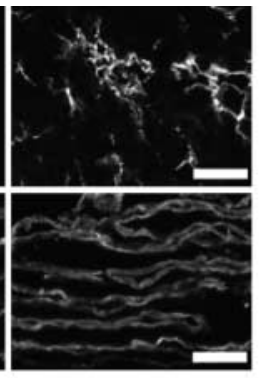

$\mathrm{Bgn}^{-/-} \mathrm{Dcn}^{+/+}$

B
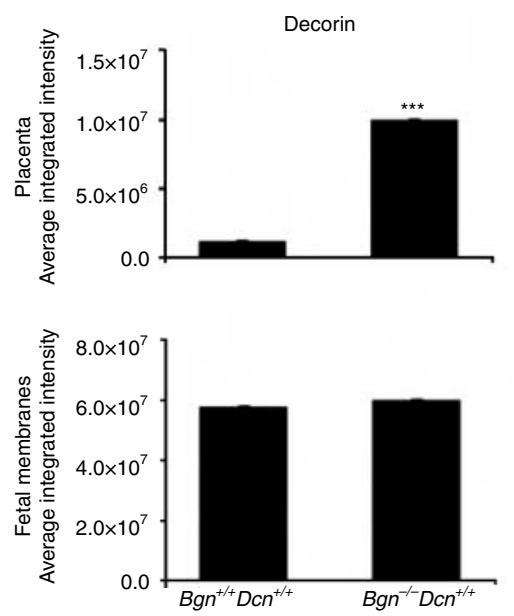

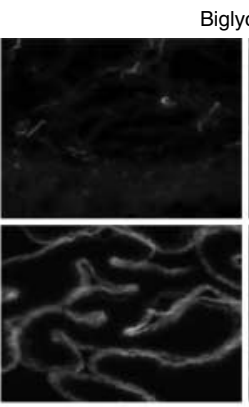

$\mathrm{Bgn}^{+/+} \mathrm{Dcn}^{+/+}$
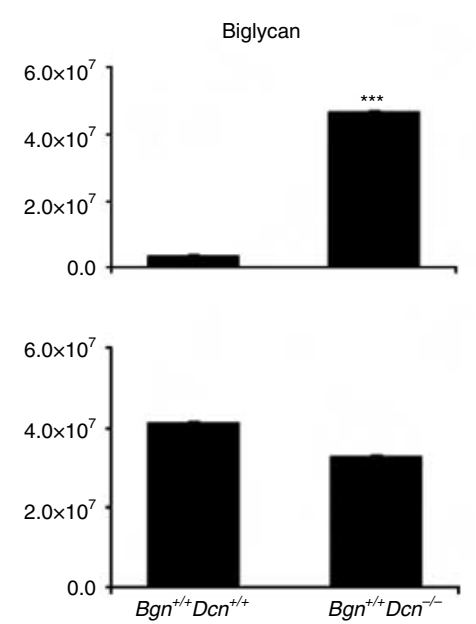

Figure 4 (A) Immunohistochemical comparison of biglycan expression in wild-type and decorin homozygous null mutant mouse placenta and fetal membranes as well as decorin expression in wildtype and biglycan homozygous null mutant mouse placenta and fetal membranes. There is increased biglycan signal in the absence of decorin and increased decorin signal in the absence of biglycan in the placenta. No compensatory upregulation is noted in the fetal membranes. Placenta decorin staining $10 \times$, scale bar $500 \mu \mathrm{m}$. All others $20 \times$, scale bar $100 \mu \mathrm{m}$. The photomicrographs are representative of one placenta each from three pregnant females per experimental group. (B) Quantitative analysis of immunofluorescence showing a significant increase in decorin signal in the absence of biglycan $(* * * P<0.001)$ and a significant increase in biglycan signal in the absence of decorin $(* * * P<0.001)$ in the placenta but no difference in fetal membranes, analyzed by Student's $t$-test. The graphs are representative of 5-15 images from one placenta each from three pregnant females per experimental group. Error bars=s.E.M. Bgn, biglycan; Dcn, decorin. are present in litters of $\mathrm{Bgn}^{+/-} \mathrm{DCn}^{+/-}$females mated with $\mathrm{Bgn}^{-/ 0} \mathrm{DCn} \mathrm{n}^{+/-}$males at P1, we suspect that the same mechanisms that lead to low birth weight also lead to late fetal or early postnatal loss. The genotype of the placenta and fetal membranes reflects that of the fetus and not of the dam, emphasizing the fact that it is the complete absence of both biglycan and decorin in the fetus that is associated with significant growth restriction and not the maternal genotype $\left(\mathrm{Bgn}^{+/-} \mathrm{DCn}^{+/-}\right)$, which is identical for all littermates.

Beyond their structural roles, both biglycan and decorin have been implicated in a host of signaling pathways that may provide insight into their mechanisms of action in the placenta leading to in utero growth restriction as well as in fetal membranes leading to preterm birth.

Among these pathways are the TGFB, BMP, SMAD, and WNT-WISP pathways. TGFB signals via the SMAD family of intracellular transcription factors, demonstrates cross talk with the WNT-WISP pathway at a number of levels and plays a crucial role in embryonic development as well as a multitude of other processes (Guo \& Wang 2009). Biglycan and decorin interact with these pathways in a number of ways. Their differential expression is regulated by these growth factors (reviewed in Kinsella et al. (2004)). They both bind TGFB and decorin modulates its activity via negative feedback mechanisms (Bassols \& Massague 1988, Okuda et al. 1990, Yamaguchi et al. 1990, Border et al. 1992), whereas both biglycan and decorin bind WISP1 and modulate its activity (Desnoyers et al. 2001). Also, decorin plays a role in placental trophoblast antimigratory activity (lacob et al. 2008). Thus, the absence of both biglycan and decorin may lead to dysregulation of placental growth via complex and system-specific interactions within the TGFB, BMP, SMAD, and WNT-WISP pathways.

Another possible mechanism of biglycan and decorin's role in in utero growth restriction is their function in anti-coagulation. The placentae of infants with in utero growth restriction display decreased biglycan and decorin as well as thrombosis (Fuke et al. 1994, Murthi et al. 2010, Swan et al. 2010), whereas the glycosaminoglycan chains on biglycan and decorin display anti-coagulant activity (He et al. 2008). Thus, the absence of biglycan and decorin in the placenta may lead to thrombosis and fibrosis of the placenta with subsequent in utero growth restriction.

Biglycan is also a ligand for the transmembrane receptors Toll-like receptors 2 and 4, thus playing a role in inflammatory cascade activation (Schaefer et al. 2005). Via this pathway, biglycan may play a role in 


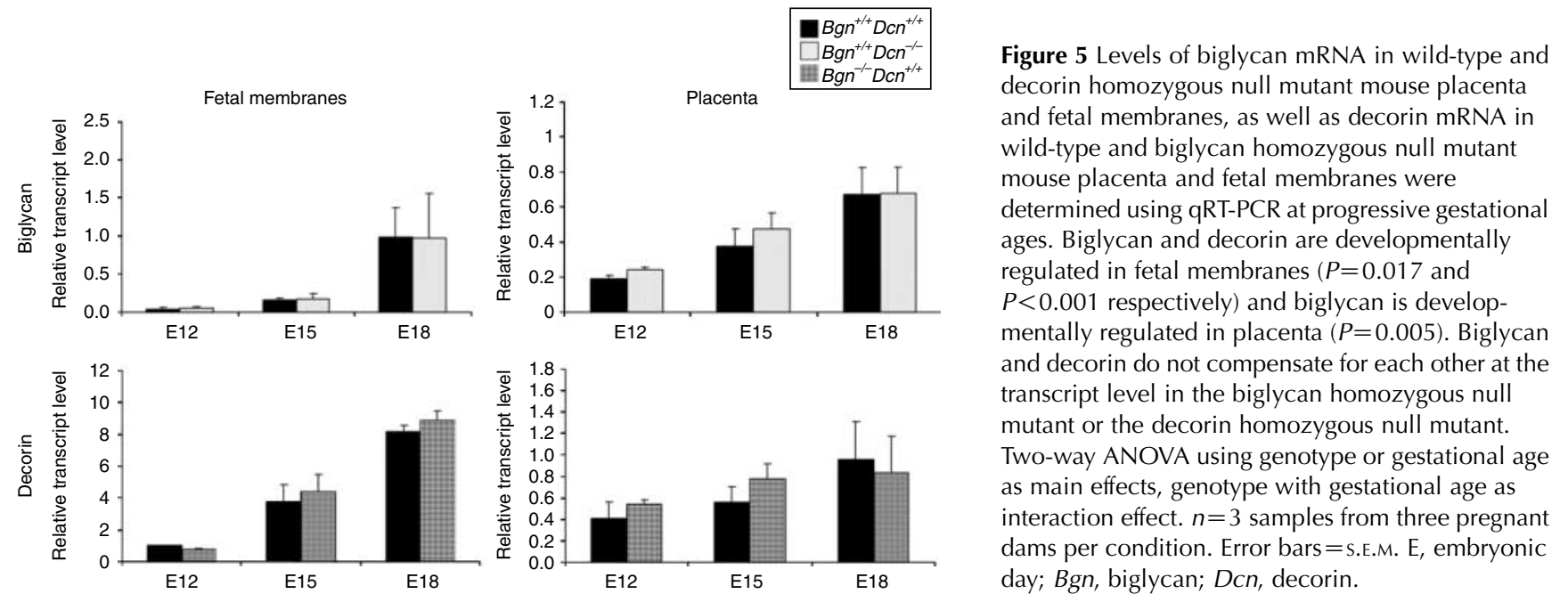

modulating an inflammatory response whose dysregulation may lead to PPROM or in utero growth restriction.

The underlying etiology of the preterm birth in our model is particularly challenging to ascertain. It is unclear whether it is the fetal genotype, the maternal genotype, or a combination of both that is causative of the phenotype. In patients with connective tissue anomalies secondary to EDS, preterm birth is caused by PPROM. Changes in collagen content and/or structure have been proposed to play a role in the pathogenesis of PPROM (Parry \& Strauss 1998). Biglycan and decorin are highly expressed in fetal membranes (Meinert et al. 2007) and bind collagens in the extracellular matrix (Brown \& Vogel 1989, Hildebrand et al. 1994, Wiberg et al. 2001, 2003, Chen et al. 2004, Hayashi et al. 2005, Moreno et al. 2005). Thus, we hypothesize that the complete absence of both biglycan and decorin leads to decreased fetal membrane tensile strength and ultimately PPROM as the mechanism for the preterm birth observed in the biglycan homozygous/ decorin homozygous null mutant $\left(\mathrm{Bgn}^{-1-} \mathrm{DCn}^{-1-}\right)$ dams. In addition, biglycan and decorin are differentially expressed in fetal membranes (Meinert et al. 2007). Decorin decreases prior to parturition as membrane tensile strength decreases, although biglycan increases (Meinert et al. 2007). Thus, given biglycan's role in signaling in other tissues (Chen et al. 2004, Moreno et al. 2005, Schaefer et al. 2005), it is possible that biglycan has a functional role beyond its biophysical, structural role in fetal membranes.

Another possible mechanism of biglycan and decorin's role in PPROM involves their interactions with MMPs. MMPs are proteases that are involved in extracellular matrix remodeling associated with various physiological and pathological processes (Malemud 2006). A compelling body of evidence suggests that MMPs play a significant role in the pathogenesis of PPROM (Menon \& Fortunato 2004, Vadillo-Ortega \&
Estrada-Gutierrez 2005). The mechanism by which MMPs are thought to contribute to the pathogenesis of PPROM is through the degradation of the extracellular matrix of fetal membranes. Proteoglycans and collagens are present in the fetal membranes (Parry \& Strauss 1998). The substrate affinities of MMP2, -3, -8, -9, and -13, all of which are expressed in fetal membranes, suggest that they target the various collagens as well as the proteoglycans within the fetal membrane extracellular matrix (Vadillo-Ortega \& Estrada-Gutierrez 2005). Also, decorin induces MMP1, -2, and -14 synthesis (Schonherr et al. 2001). Furthermore, biglycan as well as decorin are associated with increased expression of MMP1 (Huttenlocher et al. 1996). These observations suggest that biglycan and decorin may play a role in the process of tissue remodeling via interactions with MMPs, leading to PPROM.

Another possible mechanism of biglycan and decorin's role in PPROM involves their interactions with the TGFB, BMP, and SMAD signaling pathways described above. The TGFB, SMAD, and biglycan/ decorin signaling pathways are involved in the development of aortic aneurysms in humans, with SMAD2 levels correlating with extracellular matrix elastic fiber destruction (Gomez et al. 2009). Humans with EDS (Barabas 1972), biglycan homozygous null mutant $\left(B^{-1-} \mathrm{DCn}^{+/+}\right)$mice (Heegaard et al. 2007), and biglycan homozygous/decorin heterozygous null mutant mice $\left(\mathrm{Bgn}^{-1-} \mathrm{DCn}^{+/-}\right)$(data not shown) are at increased risk of developing aortic aneurysm and subsequent rupture. Aneurysms and subsequent aortic rupture are caused by the weakening of the vessel's connective tissue extracellular matrix; similarly, PPROM is caused by breakdown of fetal membrane connective tissue extracellular matrix. Biglycan is decreased in human aortic aneurysms and decorin is dyslocalized (Tamarina et al. 1998, Theocharis \& Karamanos 2002), whereas biglycan is increased and decorin is decreased in 
ruptured fetal membranes (Meinert et al. 2007). TGFB, via its interactions with biglycan and decorin (reviewed in Kinsella et al. (2004)), is involved in the regulation of collagens and MMPs (Ignotz \& Massague 1986, Ma et al. 1999). Aortic rupture and rupture of fetal membranes display pathophysiological similarities that involve biglycan and decorin. Thus, the TGFB, SMAD, and biglycan/decorin signaling pathways may lead to PPROM via interactions with the MMPs and collagens (reviewed in Kinsella et al. (2004)), mirroring its role in aortic rupture pathophysiology.

Thus, we hypothesize that biglycan and decorin may play a role in reproduction by playing a significant role in maintaining the structural integrity of the fetal membrane extracellular matrix. Dysregulation by physiologic and/or pathologic processes via any or all of the aforementioned structural/signaling pathways may lead to tissue remodeling and subsequently to preterm premature rupture of membranes.

Nonetheless, based on the present data, we cannot rule out mechanisms other than PPROM leading to preterm birth. The maternal biglycan homozygous/decorin homozygous null mutant $\left(\mathrm{Bgn}^{-/-} \mathrm{DCn}^{-1-}\right)$ genotype may cause uterine or cervical pathology leading to the preterm birth. Alternately, the genotype of the individual pups and their placenta and fetal membranes, be that biglycan homozygous/decorin homozygous null mutant $\left(\mathrm{Bgn}^{-1-} \mathrm{DCn^{- } -}\right)$ or others, may be leading to placental pathologies such as preeclampsia or to fetal membrane pathologies such as PPROM or chorioamnionitis, which then lead to preterm birth. Possibly, it is the interaction of both maternal and fetal gestational tissue pathology, each with its own individual genotype, which is leading to the observed preterm birth. Given the genetically complex nature of the maternal and fetal genotypes and thus the variety of possible genotype combinations that contribute to the gestational outcome, future experiments need to be thoughtfully geared toward controlling for the contribution of maternal and individual pup genetics on preterm birth outcomes. This would provide valuable insight into the mechanism by which biglycan and decorin contribute to the attainment of full-term gestation.

In summary, we present a novel gene dose-dependent mouse model of genetic preterm birth and in utero growth restriction and show that biglycan and decorin exhibit compensatory mechanisms. In addition to preterm birth, the loss of biglycan and decorin results in perinatal fetal loss and low birth weight. Thus, this model presents novel targets for preventive or therapeutic manipulation of preterm birth. We hope that future experiments with this model will allow for mechanistic insight into the initiators of preterm birth, as well as the discrete roles of both biglycan and decorin in gestation.

\section{Materials and Methods}

\section{Mouse nomenclature}

Biglycan homozygous null mutant: $\mathrm{Bgn}^{-/-} \mathrm{DCn}^{+/+}$female; $\mathrm{Bgn}^{-/ 0} \mathrm{DCn}^{+/+}$male.

Decorin homozygous null mutant: $\mathrm{Bgn}^{+/+} \mathrm{Dcn}^{-/-}$female; $\mathrm{Bgn}^{+/ 0} \mathrm{Dcn}^{-/-}$male.

Biglycan heterozygous/decorin heterozygous null mutant: $\mathrm{Bgn}^{+/-} \mathrm{Dcn}^{+/-}$female.

Biglycan heterozygous/decorin homozygous null mutant: $\mathrm{Bgn}^{+/-} \mathrm{DCn}^{-/-}$female.

Biglycan homozygous/decorin heterozygous null mutant: $\mathrm{Bgn}^{-/-} \mathrm{Dcn}^{+/-}$female; $\mathrm{Bgn}^{-/ 0} \mathrm{Dcn}^{+/-}$male.

Biglycan homozygous/decorin homozygous null mutant: $\mathrm{Bgn}^{-1-} \mathrm{Dcn}^{-1-}$ female; $\mathrm{Bgn}^{-10} \mathrm{Dcn}^{-1-}$ male.

\section{Mouse husbandry}

C3H wild-type mice were purchased from Jackson Laboratories (Bar Harbor, ME, USA). A biglycan homozygous null mutant breeding pair (generated by Marian Young (Xu et al. 1998)) was a gift from Justin Fallon. A decorin heterozygous null mutant $\left(\mathrm{Bgn}^{+/+} \mathrm{DCn}^{+/-}\right)$breeding pair was mated to the birth of homozygous pups, which were then bred to establish the decorin homozygous null mutant colony. Mice were housed under standard conditions. A biglycan homozygous null mutant female was crossed with a decorin homozygous null mutant male to establish breeding pairs in which the females were heterozygous for both biglycan and decorin (biglycan heterozygous/decorin heterozygous null mutant: Bgn ${ }^{+/-}$ $\mathrm{DCn}^{+/-}$) and the males were heterozygous for decorin but homozygous knockouts for biglycan $\left(\mathrm{Bgn}^{-/ 0} \mathrm{DCn}^{+/-}\right)$, given that biglycan is an X-chromosomal gene. These pairs were mated to breed mixed genotype litters. The $\mathrm{Bgn}^{-10} \mathrm{Dcn}^{+/-}$ males were also used for mating with biglycan heterozygous/ decorin homozygous null mutant and biglycan homozygous/ decorin heterozygous null mutant females $\left(\mathrm{Bgn}^{+/-} \mathrm{DCn}^{-1-}\right.$, $\mathrm{Bgn}^{-1-} \mathrm{DCn}^{+/-}$) as well as biglycan homozygous/decorin homozygous null mutant females $\left(B g n^{-1-} D c n^{-1-}\right)$. We chose this breeding strategy given that our observations demonstrate that while males with at least one SLRP allele have mating success that is not significantly different from the wild type, the biglycan homozygous/decorin homozygous null mutant male $\left(\mathrm{Bgn}^{-/ 0} \mathrm{DCn}^{-1-}\right)$ is not capable of producing a pregnancy (data not shown). Breeding pairs were set up in the evening. Plugs were checked the following morning and every morning thereafter. The day of the plug was defined as EO. Cages were observed each morning for litters. The morning that a litter was observed was defined as the day of birth. Pairs were set up for mating at 5-7 weeks of age. A subgroup of wild-type, biglycan homozygous null mutant and decorin homozygous null mutant pregnant dams were killed at E12, E15, and E18. Litter size as well as viable and nonviable embryo numbers were recorded.

Data was collected on the number of days of mating to copulatory plug, on the length of pregnancy from plug to birth, and on the litter size. Pups were weighed at P1. Mixed genotype litters were tattooed for identification after weighing and then tail snipped at P4-P7 for genotyping. A subgroup of 
the biglycan heterozygous/decorin heterozygous null mutant pregnant dams $\left(\mathrm{Bgn}^{+/} \mathrm{DCn}^{+-}\right)$was killed at E18. Pup and placental wet weight as well as litter size were recorded. Pups were tail snipped for genotyping. IACUC approval was obtained.

\section{Genotyping}

A $3 \mathrm{~mm}$ tail biopsy specimen was obtained for each pup within a mixed genotype litter at P4-P7 or at weaning. Genomic DNA was extracted from each tail biopsy sample using the High Pure PCR Template Preparation Kit (Roche). PCR was performed to identify the decorin and biglycan alleles using the Taq DNA Polymerase kit (New England Biolabs, Ipswich, MA, USA) and the PTC-200 thermal cycler. The PCR product was run on a $1.8 \% \mathrm{w} / \mathrm{v}$ agarose gel to visualize the following diagnostic bands. The decorin PCR produced bands of $161 \mathrm{bp}$ for the wild-type allele and $238 \mathrm{bp}$ for the knockout allele. The biglycan PCR produced bands of 212 and $310 \mathrm{bp}$ for the wild-type and knockout alleles respectively.

\section{RNA and CDNA preparation}

Wild-type, biglycan homozygous null mutant and decorin homozygous null mutant mouse placenta and fetal membranes were dissected at three prenatal time points (E12, E15, and E18) in $0.1 \mathrm{~mol} / \mathrm{l} \mathrm{PBS}, \mathrm{pH} 7.4$, snap-frozen in liquid nitrogen, and stored at $-80^{\circ} \mathrm{C}$. RNA extraction was performed using the Trizol method (Invitrogen). Genomic DNA was removed by incubating the RNA sample with DNase I (Invitrogen) for 30 min at $37^{\circ} \mathrm{C}$ with subsequent RNA re-extraction with Trizol. The purified RNA was converted to cDNA using the Superscript III First-Strand Synthesis System Kit (Invitrogen).

\section{Quantitative PCR analysis}

qPCRs were performed on the ABI PRISM 7000 real-time thermocycler (Applied Biosystems, Foster City, CA, USA) and on the Eppendorf Mastercycler epgradient S (Eppendorf, Hamburg, Germany) using the SYBR-Green method (Invitrogen). Primers were designed using Primer-Blast primer design software (National Library of Medicine, Bethesda, MD, USA). Gapdh was used as a normalizer. Melting point analysis of the product was performed to ensure the absence of alternative products or primer dimers. Data analysis was performed using the comparative $C_{\mathrm{t}}$ method with a validation experiment. qPCR analysis was performed in triplicate, $n=3$ samples from three dams per genotype.

\section{Primer sequences}

qPCR biglycan: forward ATTGCCCTACCCAGAACTTGAC, reverse GCAGAGTATGAACCCTTTCCTG; qPCR decorin: forward TTCCTACTCGGCTGTGAGTC, reverse AAGTTGAATGGCAGAACGC; qPCR Gapdh: forward CTCACAATTTCCATCCCAGAC, reverse TTTTTGGGTGCAGCGAAC; biglycan genotyping PCR wild-type allele: forward TGATGAGGAGGCTTCAGGTT, reverse GCAGTGTGGTGTCAGGTGAG; biglycan genotyping PCR knockout allele, forward TGTGGCTACTCACCTTGCTG, reverse GCCAGAGGCCACTTGTGTAG; decorin genotyping PCR allele: forward CCTTCTGGCACAAGTCTCTTGG, decorin genotyping PCR wild-type allele reverse TCGAAGATGACACTGGCATCGG; decorin genotyping PCR knockout allele: reverse TGGATGTGGAATGTGTGCGAG. All primers were provided by Invitrogen.

\section{Immunohistochemistry}

Wild-type, biglycan homozygous null mutant and decorin homozygous null mutant mouse placenta and fetal membranes were dissected at three prenatal time points (E12, E15, and E18) in $0.1 \mathrm{~mol} / \mathrm{IPBS}, \mathrm{pH} 7.4$. The specimens were then flash frozen in isopentane and stored at $-80^{\circ} \mathrm{C}$. The frozen tissue was cryostat sectioned to $10 \mu \mathrm{m}$ thickness, mounted on slides, and stored at $-20^{\circ} \mathrm{C}$. Sections were fixed in $1 \% \mathrm{v} / \mathrm{v}$ paraformaldehyde and stained with primary antibodies using the mouse on mouse immunostaining kit for MAbs (Vector Laboratories, Burlingame, CA, USA), then incubated with primary antibody overnight at $4{ }^{\circ} \mathrm{C}$ and with secondary antibody for $30 \mathrm{~min}$ at room temperature. Sections were mounted with Vectashield mounting medium with DAPI (Vector Laboratories). Fluorescence microscopy to evaluate the samples was performed using an inverted stage Nikon Eclipse TE2000-E microscope equipped with epifluorescent filters and a Nikon Plan Apo $20 \times$ and $40 \times$ and a Plan Fluor $10 \times$ objective lens (Yokohama, Japan). Images were acquired using a Coolsnap HQ cooled CCD camera (Roper Scientific, Ottobrunn, Germany) and MetaVue software (Molecular Devices, Downingtown, PA, USA). Experiments were repeated three times with tissue samples from three dams per genotype.

\section{Antibodies}

The antibodies used were polyclonal rabbit anti-mouse biglycan antibody LF-159 (a gift from Larry Fisher) and polyclonal goat anti-mouse decorin antibody (R\&D Systems, Minneapolis, MN, USA). Secondary antibody labeling was performed with goat anti-rabbit $\lg \mathrm{G}$ conjugated to Alexa 488 (Invitrogen) for biglycan and rabbit anti-goat IgG conjugated to CY3 (Sigma-Aldrich) for decorin. Mouse IgG and rabbit IgG (Vector Laboratories) were used as controls.

\section{Quantitative immunofluorescence analysis}

Images of tissue sections were analyzed as follows: 5-15 images per sample were collected using constant exposure and microscope settings. Images were analyzed using MetaVue software (Molecular Devices). Image sets were thresholded to remove background and the integrated intensity was calculated for each image and the average was calculated for each sample. Error bars represent S.E.M. For both placenta and fetal membranes, decorin immunofluorescence of wild type was compared with the biglycan homozygous null mutant and biglycan immunofluorescence of wild type was compared with the decorin homozygous null mutant. Image analysis was repeated on placenta and fetal membrane samples from three pregnant females per genotype and antibody. 


\section{Statistical analysis}

The occurrence of preterm births across genotypes was analyzed using the $\chi^{2}$ test, and the relationship of preterm birth to number of alleles was analyzed using the $\chi^{2}$ for linear trend test. Analysis of gestational length and prenatal litter size was conducted using one-way ANOVA with a planned contrast of all genotypes versus wild type using the Holm-Sidak method. Comparison of the number of biglycan homozygous/ decorin homozygous null mutant pups with the Mendelian expected number was done using the one-way $\chi^{2}$ test. Analysis of prenatal and postnatal pup weights and placental weights was conducted with mixed models, with genotype as a fixed effect and litter as a random effect, in order to control for the correlation of measures within litters. Specific planned contrasts were also done in these models, comparing biglycan homozygous/decorin homozygous null mutant pups to all other genotypes. Analysis of E18 versus P1 litter size as well as average integrated fluorescence intensity was performed using Student's $t$-test. Analysis of relative ratios was done with twoway ANOVA using genotype and gestational age as main effects, as well as a genotype $\times$ gestational age interaction effect. Analysis of days to plug formation was done with Cox regression with robust sandwich covariance matrix estimate to adjust for clustered data per mother. Analysis of progression of plug to pregnancy was done with Poisson regression with generalized estimating equations to adjust for correlated observations. Analysis of resorbed implantation sites was done with negative binomial regression. The software used for these analyses was Sigmaplot version 11.0 and SAS version 9.1 (SAS, Cary, NC, USA).

\section{Supplementary data}

This is linked to the online version of the paper at http://dx.doi. org/10.1530/REP-10-0387.

\section{Declaration of interest}

The authors declare that there is no conflict of interest that could be perceived as prejudicing the impartiality of the research reported.

\section{Funding}

This work was supported by NICHD 1K08HD054676, NCRR 5P20RR018728-08, and NCI R01CA39481.

\section{Acknowledgements}

We thank Larry Fisher for the generous gift of the anti-biglycan antibodies; Justin Fallon for generously providing a biglycan knockout mouse breeding pair; and Sunil Shaw, Joseph Bliss, and Edward Chien for valuable discussions.

\section{References}

Ameye L \& Young MF 2002 Mice deficient in small leucine-rich proteoglycans: novel in vivo models for osteoporosis, osteoarthritis, Ehlers-Danlos syndrome, muscular dystrophy, and corneal diseases. Glycobiology 12 107R-116R. (doi:10.1093/glycob/cwf065)

Ananth CV \& Vintzileos AM 2006 Epidemiology of preterm birth and its clinical subtypes. Journal of Maternal-Fetal \& Neonatal Medicine 19 773-782. (doi:10.1080/14767050600965882)

Arikat S, Novince RW, Mercer BM, Kumar D, Fox JM, Mansour JM \& Moore JJ 2006 Separation of amnion from choriodecidua is an integral event to the rupture of normal term fetal membranes and constitutes a significant component of the work required. American Journal of Obstetrics and Gynecology 194 211-217. (doi:10.1016/j.ajog.2005. 06.083)

Barabas AP 1966 Ehlers-Danlos syndrome: associated with prematurity and premature rupture of foetal membranes; possible increase in incidence. BMJ 5515 682-684. (doi:10.1136/bmj.2.5515.682)

Barabas AP 1972 Vascular complications in the Ehlers-Danlos syndrome, with special reference to the "arterial type" or Sack's syndrome. Journal of Cardiovascular Surgery 13 160-167.

Bassols A \& Massague J 1988 Transforming growth factor beta regulates the expression and structure of extracellular matrix chondroitin/ dermatan sulfate proteoglycans. Journal of Biological Chemistry 263 3039-3045.

Bizargity P, Del Rio R, Phillippe M, Teuscher C \& Bonney EA 2009 Resistance to lipopolysaccharide-induced preterm delivery mediated by regulatory T cell function in mice. Biology of Reproduction 80 874-881. (doi:10.1095/biolreprod.108.074294)

Border WA, Noble NA, Yamamoto T, Harper JR, Yamaguchi Y, Pierschbacher MD \& Ruoslahti E 1992 Natural inhibitor of transforming growth factor-beta protects against scarring in experimental kidney disease. Nature 360 361-364. (doi:10.1038/360361a0)

Bowe MA, Mendis DB \& Fallon JR 2000 The small leucine-rich repeat proteoglycan biglycan binds to alpha-dystroglycan and is upregulated in dystrophic muscle. Journal of Cell Biology 148 801-810. (doi:10.1083/ jcb.148.4.801)

Brown DC \& Vogel KG 1989 Characteristics of the in vitro interaction of a small proteoglycan (PG II) of bovine tendon with type I collagen. Matrix 9 468-478.

Chen XD, Fisher LW, Robey PG \& Young MF 2004 The small leucine-rich proteoglycan biglycan modulates BMP-4-induced osteoblast differentiation. FASEB Journal 18 948-958. (doi:10.1096/fj.03-0899com)

Chen CP, Chang SC \& Vivian Yang WC 2007 High glucose alters proteoglycan expression and the glycosaminoglycan composition in placentas of women with gestational diabetes mellitus and in cultured trophoblasts. Placenta 28 97-106. (doi:10.1016/j.placenta.2006.02.009)

Corsi A, Xu T, Chen XD, Boyde A, Liang J, Mankani M, Sommer B, Iozzo RV, Eichstetter I, Robey PG et al. 2002 Phenotypic effects of biglycan deficiency are linked to collagen fibril abnormalities, are synergized by decorin deficiency, and mimic Ehlers-Danlos-like changes in bone and other connective tissues. Journal of Bone and Mineral Research 17 1180-1189. (doi:10.1359/jbmr.2002.17.7.1180)

Danielson KG, Baribault H, Holmes DF, Graham H, Kadler KE \& lozzo RV 1997 Targeted disruption of decorin leads to abnormal collagen fibril morphology and skin fragility. Journal of Cell Biology 136 729-743. (doi:10.1083/jcb.136.3.729)

Desnoyers L, Arnott D \& Pennica D 2001 WISP-1 binds to decorin and biglycan. Journal of Biological Chemistry 276 47599-47607. (doi:10. 1074/jbc.M108339200)

El Khwad M, Pandey V, Stetzer B, Mercer BM, Kumar D, Moore RM, Fox J, Redline RW, Mansour JM \& Moore JJ 2006 Fetal membranes from term vaginal deliveries have a zone of weakness exhibiting characteristics of apoptosis and remodeling. Journal of the Society for Gynecologic Investigation 13 191-195. (doi:10.1016/j.jsgi.2005.12.010)

Fuke Y, Aono T, Imai S, Suehara N, Fujita T \& Nakayama M 1994 Clinical significance and treatment of massive intervillous fibrin deposition associated with recurrent fetal growth retardation. Gynecologic and Obstetric Investigation 38 5-9. (doi:10.1159/000292434)

Gogiel T \& Jaworski S 2000 Proteoglycans of human umbilical cord arteries. Acta Biochimica Polonica 47 1081-1091. 
Gogiel T, Bankowski E \& Jaworski S 2003 Proteoglycans of Wharton's jelly. International Journal of Biochemistry \& Cell Biology 35 1461-1469. (doi:10.1016/S1357-2725(03)00128-6)

Gomez D, Al Haj Zen A, Borges LF, Philippe M, Gutierrez PS, Jondeau G, Michel JB \& Vranckx R 2009 Syndromic and non-syndromic aneurysms of the human ascending aorta share activation of the Smad2 pathway. Journal of Pathology 218 131-142. (doi:10.1002/path.2516)

Guo X \& Wang XF 2009 Signaling cross-talk between TGF-beta/BMP and other pathways. Cell Research 19 71-88. (doi:10.1038/cr.2008.302)

Hayashi Y, Liu CY, Jester JJ, Hayashi M, Wang IJ, Funderburgh JL, Saika S, Roughley PJ, Kao CW \& Kao WW 2005 Excess biglycan causes eyelid malformation by perturbing muscle development and TGF-alpha signaling. Developmental Biology 277 222-234. (doi:10.1016/j.ydbio. 2004.09.022)

He L, Giri TK, Vicente CP \& Tollefsen DM 2008 Vascular dermatan sulfate regulates the antithrombotic activity of heparin cofactor II. Blood 111 4118-4125. (doi:10.1182/blood-2007-12-127928)

Heegaard AM, Corsi A, Danielsen CC, Nielsen KL, Jorgensen HL, Riminucci M, Young MF \& Bianco P 2007 Biglycan deficiency causes spontaneous aortic dissection and rupture in mice. Circulation 115 2731-2738. (doi:10.1161/CIRCULATIONAHA.106.653980)

Hermanns-Le T, Pierard G \& Quatresooz P 2005 Ehlers-Danlos-like dermal abnormalities in women with recurrent preterm premature rupture of fetal membranes. American Journal of Dermatopathology 27 407-410. (doi:10.1097/01.dad.0000175529.42615.42)

Hildebrand A, Romaris M, Rasmussen LM, Heinegard D, Twardzik DR, Border WA \& Ruoslahti E 1994 Interaction of the small interstitial proteoglycans biglycan, decorin and fibromodulin with transforming growth factor beta. Biochemical Journal 302 527-534.

Hjelm AM, Barchan K, Malmstrom A \& Ekman-Ordeberg GE 2002 Changes of the uterine proteoglycan distribution at term pregnancy and during labour. European Journal of Obstetrics, Gynecology, and Reproductive Biology 100 146-151. (doi:10.1016/S0301-2115(01)00476-6)

lacob D, Cai J, Tsonis M, Babwah A, Chakraborty C, Bhattacharjee RN \& Lala PK 2008 Decorin-mediated inhibition of proliferation and migration of the human trophoblast via different tyrosine kinase receptors. Endocrinology 149 6187-6197. (doi:10.1210/en.2008-0780)

Ignotz RA \& Massague J 1986 Transforming growth factor-beta stimulates the expression of fibronectin and collagen and their incorporation into the extracellular matrix. Journal of Biological Chemistry 261 4337-4345.

Iozzo RV \& Murdoch AD 1996 Proteoglycans of the extracellular environment: clues from the gene and protein side offer novel perspectives in molecular diversity and function. FASEB Journal 10 598-614.

Kinsella MG, Bressler SL \& Wight TN 2004 The regulated synthesis of versican, decorin, and biglycan: extracellular matrix proteoglycans that influence cellular phenotype. Critical Reviews in Eukaryotic Gene Expression 14 203-234. (doi:10.1615/CritRevEukaryotGeneExpr.v14.i3.40)

Kresse H, Rosthoj S, Quentin E, Hollmann J, Glossl J, Okada S \& Tonnesen T 1987 Glycosaminoglycan-free small proteoglycan core protein is secreted by fibroblasts from a patient with a syndrome resembling progeroid. American Journal of Human Genetics 41 436-453.

Ma C, Tarnuzzer RW \& Chegini N 1999 Expression of matrix metalloproteinases and tissue inhibitor of matrix metalloproteinases in mesothelial cells and their regulation by transforming growth factorbeta1. Wound Repair and Regeneration 7 477-485. (doi:10.1046/j. 1524-475X.1999.00477.x)

Malemud CJ 2006 Matrix metalloproteinases (MMPs) in health and disease: an overview. Frontiers in Bioscience 11 1696-1701. (doi:10.2741/1915)

Meinert M, Eriksen GV, Petersen AC, Helmig RB, Laurent C, Uldbjerg N \& Malmstrom A 2001 Proteoglycans and hyaluronan in human fetal membranes. American Journal of Obstetrics and Gynecology 184 679-685. (doi:10.1067/mob.2001.110294)

Meinert M, Malmstrom A, Tufvesson E, Westergren-Thorsson G, Petersen AC, Laurent C, Uldbjerg N \& Eriksen GV 2007 Labour induces increased concentrations of biglycan and hyaluronan in human fetal membranes. Placenta 28 482-486. (doi:10.1016/j.placenta.2006.09.006)

Menon R \& Fortunato SJ 2004 The role of matrix degrading enzymes and apoptosis in rupture of membranes. Journal of the Society for Gynecologic Investigation 11 427-437. (doi:10.1016/j.jsgi.2004. 04.001)
Moore RM, Mansour JM, Redline RW, Mercer BM \& Moore JJ 2006 The physiology of fetal membrane rupture: insight gained from the determination of physical properties. Placenta 27 1037-1051. (doi:10. 1016/j.placenta.2006.01.002)

Moreno M, Munoz R, Aroca F, Labarca M, Brandan E \& Larrain J 2005 Biglycan is a new extracellular component of the Chordin-BMP4 signaling pathway. EMBO Journal 24 1397-1405. (doi:10.1038/sj. emboj.7600615)

Murthi P, Faisal FA, Rajaraman G, Stevenson J, Ignjatovic V, Monagle PT, Brennecke SP \& Said JM 2010 Placental biglycan expression is decreased in human idiopathic fetal growth restriction. Placenta 31 712-717. (doi:10.1016/j.placenta.2010.05.009)

Okuda S, Languino LR, Ruoslahti E \& Border WA 1990 Elevated expression of transforming growth factor-beta and proteoglycan production in experimental glomerulonephritis. Possible role in expansion of the mesangial extracellular matrix. Journal of Clinical Investigation $\mathbf{8 6}$ 453-462. (doi:10.1172/JCI114731)

Parry S \& Strauss JF III 1998 Premature rupture of the fetal membranes. New England Journal of Medicine 338 663-670. (doi:10.1056/NEJM 199803053381006)

Quentin E, Gladen A, Roden L \& Kresse H 1990 A genetic defect in the biosynthesis of dermatan sulfate proteoglycan: galactosyltransferase I deficiency in fibroblasts from a patient with a progeroid syndrome. PNAS 87 1342-1346. (doi:10.1073/pnas.87.4.1342)

Reed CC \& lozzo RV 2002 The role of decorin in collagen fibrillogenesis and skin homeostasis. Glycoconjugate Journal 19 249-255. (doi:10. 1023/A:1025383913444)

Robertson SA, Skinner RJ \& Care AS 2006 Essential role for IL-10 in resistance to lipopolysaccharide-induced preterm labor in mice. Journal of Immunology 177 4888-4896.

Romero R, Mazor M, Munoz H, Gomez R, Galasso M \& Sherer DM 1994 The preterm labor syndrome. Annals of the New York Academy of Sciences 734 414-429. (doi:10.1111/j.1749-6632.1994.tb21771.x)

Roop KA \& Brost BC 1999 Abnormal presentation in labor and fetal growth of affected infants with type III Ehlers-Danlos syndrome. American Journal of Obstetrics and Gynecology 181 752-753. (doi:10.1016/ S0002-9378(99)70524-7)

Sanches JC, Jones CJ, Aplin JD, lozzo RV, Zorn TM \& Oliveira SF 2010 Collagen fibril organization in the pregnant endometrium of decorindeficient mice. Journal of Anatomy 216 144-155. (doi:10.1111/j.14697580.2009.01170.x)

San Martin S \& Zorn TM 2003 The small proteoglycan biglycan is associated with thick collagen fibrils in the mouse decidua. Cellular and Molecular Biology 49 673-678.

San Martin S, Soto-Suazo M, De Oliveira SF, Aplin JD, Abrahamsohn P \& Zorn TM 2003 Small leucine-rich proteoglycans (SLRPs) in uterine tissues during pregnancy in mice. Reproduction 125 585-595. (doi:10. 1530/rep.0.1250585)

Schaefer L \& lozzo RV 2008 Biological functions of the small leucine-rich proteoglycans: from genetics to signal transduction. Journal of Biological Chemistry 283 21305-21309. (doi:10.1074/jbc.R800020200)

Schaefer L, Babelova A, Kiss E, Hausser HJ, Baliova M, Krzyzankova M, Marsche G, Young MF, Mihalik D, Gotte M et al. 2005 The matrix component biglycan is proinflammatory and signals through Toll-like receptors 4 and 2 in macrophages. Journal of Clinical Investigation $\mathbf{1 1 5}$ 2223-2233. (doi:10.1172/JCI23755)

Schonherr E, Schaefer L, O'Connell BC \& Kresse H 2001 Matrix metalloproteinase expression by endothelial cells in collagen lattices changes during co-culture with fibroblasts and upon induction of decorin expression. Journal of Cellular Physiology 187 37-47. (doi:10.1002/ 1097-4652(2001)9999:9999 < ::AID-JCP1048>3.0.CO;2-W)

Steer P 2005 The epidemiology of preterm labour. BJOG : an International Journal of Obstetrics and Gynaecology 112 (Supplement 1) 1-3. (doi:10. 1111/J.1471-0528.2005.00575.X)

Svensson L, Aszodi A, Reinholt FP, Fassler R, Heinegard D \& Oldberg A 1999 Fibromodulin-null mice have abnormal collagen fibrils, tissue organization, and altered lumican deposition in tendon. Journal of Biological Chemistry 274 9636-9647. (doi:10.1074/jbc.274.14.9636)

Swan BC, Murthi P, Rajaraman G, Pathirage NA, Said JM, Ignjatovic V, Monagle PT \& Brennecke SP 2010 Decorin expression is decreased in human idiopathic fetal growth restriction. Reproduction, Fertility, and Development 22 949-955. (doi:10.1071/RD09240) 
Tamarina NA, Grassi MA, Johnson DA \& Pearce WH 1998 Proteoglycan gene expression is decreased in abdominal aortic aneurysms. Journal of Surgical Research 74 76-80. (doi:10.1006/jsre.1997.5201)

Thaxton JE, Romero R \& Sharma S 2009 TLR9 activation coupled to IL-10 deficiency induces adverse pregnancy outcomes. Journal of Immunology 183 1144-1154. (doi:10.4049/jimmunol.0900788)

Theocharis AD \& Karamanos NK 2002 Decreased biglycan expression and differential decorin localization in human abdominal aortic aneurysms. Atherosclerosis 165 221-230. (doi:10.1016/S0021-9150(02)00231-9)

Vadillo-Ortega F \& Estrada-Gutierrez G 2005 Role of matrix metalloproteinases in preterm labour. BJOG : an International Journal of Obstetrics and Gynaecology 112 (Supplement 1) 19-22. (doi:10.1111/j.14710528.2005.00579.x)

Valiyaveettil M, Achur RN, Muthusamy A \& Gowda DC 2004 Characterization of chondroitin sulfate and dermatan sulfate proteoglycans of extracellular matrices of human umbilical cord blood vessels and Wharton's jelly. Glycoconjugate Journal 21 361-375. (doi:10.1023/ B:GLYC.0000046276.77147.b2)

Wadhwa S, Embree MC, Bi Y \& Young MF 2004 Regulation, regulatory activities, and function of biglycan. Critical Reviews in Eukaryotic Gene Expression 14 301-315. (doi:10.1615/CritRevEukaryotGeneExpr. v14.i4.50)

Wang X, Hagberg H, Mallard C, Zhu C, Hedtjarn M, Tiger CF, Eriksson K, Rosen A \& Jacobsson B 2006 Disruption of interleukin-18, but not interleukin-1, increases vulnerability to preterm delivery and fetal mortality after intrauterine inflammation. American Journal of Pathology 169 967-976. (doi:10.2353/ajpath.2006.050207)

Wiberg C, Hedbom E, Khairullina A, Lamande SR, Oldberg A, Timpl R, Morgelin M \& Heinegard D 2001 Biglycan and decorin bind close to the n-terminal region of the collagen VI triple helix. Journal of Biological Chemistry 276 18947-18952. (doi:10.1074/jbc.M100625200)

Wiberg C, Klatt AR, Wagener R, Paulsson M, Bateman JF, Heinegard D \& Morgelin M 2003 Complexes of matrilin-1 and biglycan or decorin connect collagen VI microfibrils to both collagen II and aggrecan. Journal of Biological Chemistry 278 37698-37704. (doi:10.1074/jbc. M304638200)

Xu T, Bianco P, Fisher LW, Longenecker G, Smith E, Goldstein S, Bonadio J, Boskey A, Heegaard AM, Sommer B et al. 1998 Targeted disruption of the biglycan gene leads to an osteoporosis-like phenotype in mice. Nature Genetics 20 78-82. (doi:10.1038/2477)

Xu G, Guimond MJ, Chakraborty C \& Lala PK 2002 Control of proliferation, migration, and invasiveness of human extravillous trophoblast by decorin, a decidual product. Biology of Reproduction 67 681-689. (doi:10.1095/biolreprod67.2.681)

Yamaguchi Y, Mann DM \& Ruoslahti E 1990 Negative regulation of transforming growth factor-beta by the proteoglycan decorin. Nature 346 281-284. (doi:10.1038/346281a0)

Yen JL, Lin SP, Chen MR \& Niu DM 2006 Clinical features of Ehlers-Danlos syndrome. Journal of the Formosan Medical Association 105 475-480. (doi:10.1016/S0929-6646(09)60187-X)

Young MF, Bi Y, Ameye L \& Chen XD 2002 Biglycan knockout mice: new models for musculoskeletal diseases. Glycoconjugate Journal 19 257-262. (doi:10.1023/A:1025336114352)

Zhang G, Chen S, Goldoni S, Calder BW, Simpson HC, Owens RT, McQuillan DJ, Young MF, lozzo RV \& Birk DE 2009 Genetic evidence for the coordinated regulation of collagen fibrillogenesis in the cornea by decorin and biglycan. Journal of Biological Chemistry 284 8888-8897. (doi:10.1074/jbc.M806590200)

Received 10 September 2010

First decision 18 October 2010

Revised manuscript received 13 April 2011

Accepted 18 April 2011 